\title{
Advances in Management of Intestinal Behçet's Disease: A Perspective From Gastroenterologists
}

\author{
Jae Hee Cheon, M.D., Ph.D. \\ Department of Internal Medicine and Institute of Gastroenterology, Yonsei University College of Medicine, Seoul, Korea
}

Intestinal Behçet's disease (intestinal BD) is a rare chronic inflammatory disorder of the intestine that is characterized by recurrent intestinal manifestations with other systemic features of BD. Intestinal BD is diagnosed when a typically shaped ulcer is observed in the gastrointestinal tract, and the clinical findings meet the diagnostic criteria for BD. Owing to the small number of patients, intestinal BD is easily underestimated. On the other hand, but it often requires surgical treatment because of severe complications, including intestinal perforations or massive bleeding. The same treatment strategies used for inflammatory bowel diseases, such as Crohn's disease and ulcerative colitis, are used for intestinal BD. 5-Aminosalicylic acids, corticosteroids, and immunomodulators are considered conventional therapies, but a considerable number of patients eventually become unresponsive to these pharmaceutical treatments. Recently, biologic agents, such as anti-tumor necrosis factor-alpha inhibitors, have also been suggested as a new treatment option for intestinal BD. This article reviews the pathogenesis and diagnosis of intestinal BD and the current treatment strategies that are expected to be useful for rheumatologic specialists. (J Rheum Dis 2021;28:4-16)

Key Words. Behçet's disease, Intestinal Behçet's disease, Therapeutic strategy, Anti-TNF- $\alpha$ inhibitor

\section{INTRODUCTION}

Behçet's disease $(\mathrm{BD})$ is a chronic and relapsing disorder that causes multisystemic inflammation. The disease was named after the Turkish dermatologist Hulûsi Behçet, who described BD for the first time in 1937 in patients with symptoms consisting of aphthous oral ulcers, genital ulcers, and iritis [1]. BD is rare in Western countries but is relatively prevalent in East Asia, Middle East Asia, and the Mediterranean region. In general, $\mathrm{BD}$ involves oral and genital ulcers and vascular and ocular manifestations. Occasionally, it can also occur in joints, central nervous system, and gastrointestinal tract. Intestinal BD is defined when a patient diagnosed with $\mathrm{BD}$ has prevalent intestinal symptoms and typical ulcerations observed on an endoscopic examination [2,3]. Among patients with BD, up to $5 \% \sim 10 \%$ are diagnosed with intestinal $\mathrm{BD}[4,5]$.
Similar to BD, the causes of intestinal BD are not completely understood, but genetic, immunologic, and environmental factors appear to be associated with the disease. The major symptoms of intestinal BD include abdominal pain, diarrhea, severe hematochezia, and even intestinal perforation. The symptoms and clinical courses are heterogeneous, ranging from mild to severe, and several patients show a poor prognosis. The demand for novel therapy and clinical trials is high because it is currently incurable, but relatively few studies have been conducted because of its low prevalence. Despite this limitation, clinical studies have been conducted [4]. Another aspect of intestinal BD is its similarity to inflammatory bowel disease (IBD). These two diseases share the same genetic background, pathogenesis, treatment strategies, and clinical outcomes. Hence, gastroenterologists often classified them as the same disease entity. Based on studies on the

Received : July 29, 2020, Revised : August 9, 2020, Accepted : August 10, 2020

Corresponding to : Jae Hee Cheon (iD http://orcid.org/0000-0002-2282-8904

Department of Internal Medicine and Institute of Gastroenterology, Yonsei University College of Medicine, 50-1 Yonsei-ro, Seodaemun-gu, Seoul 03722, Korea. E-mail : geniushee@yuhs.ac 
pathogenesis of immune-related diseases and clinical similarity of these two diseases, the therapeutic armamentarium for intestinal BD has been developed in accordance with that for IBD.

On the other hand, there is a critical demand for elaborate therapeutic strategies for intestinal $\mathrm{BD}$ because it frequently fails to respond to conventional therapies and is a long-standing disease requiring large amounts of medications. Moreover, BD is handled mainly by rheumatologists, whereas the gastroenterologists have more experience with patients with intestinal involvement of $\mathrm{BD}$. Accordingly, intestinal $\mathrm{BD}$, which has overlapping parts in terms of vasculitis and intestinal pathologies, requires cooperative care that can integrate expertise between rheumatology and gastroenterology under a systemic communication route. This review article provides up-to-date information for rheumatologist readers on the pathogenesis and treatment options for intestinal BD from the perspective of a gastroenterologist.

\section{MAIN SUBJECTS}

\section{Behçet's disease and intestinal Behçet's disease}

$\mathrm{BD}$ is difficult to confirm because no definitive diagnostic biomarker has been identified, and the related symptoms often develop asynchronously. Moreover, intestinal BD is more complicated because of its similarity to other illnesses, such as Crohn's disease and intestinal tuberculosis. Thus, a differential diagnosis is important for deciding the therapeutic approach for intestinal BD. The diagnostic criteria for $\mathrm{BD}$ recommended by the $\mathrm{BD}$ research committee of Japan in 1987 have been used widely and consist of four major and five minor symptoms [6]. In 1990, the International Study Group for Behçet's Disease also recommended the diagnostic criteria, which include recurrent oral ulceration and the presence of any two of the following clinical manifestations: genital ulcers, skin lesions, eye involvement, and positive pathergy reaction [7]. Traditionally, BD and intestinal BD are diagnosed based on subjective symptom assessments, which are often challenging and dependent on the clinicians' experience. For a long time, intestinal $\mathrm{BD}$ was diagnosed when $\mathrm{BD}$ patients with predominantly gastrointestinal symptoms showed intestinal ulcerations on endoscopic and radiological examinations [3].

On the other hand, a delayed diagnosis is prevalent because not all patients with intestinal BD show systemic symptoms at the time of the endoscopic examination [8] because these symptoms may appear several years after the patient's first experience of intestinal discomfort [9]. To overcome these limitations, novel diagnostic criteria have been recommended by the Korean Association for the Study of Intestinal Diseases using the modified Delphi process, which is now commonly used in clinical trials and patient treatment [10]. In accordance with these criteria, intestinal BD can be diagnosed when ulcer-

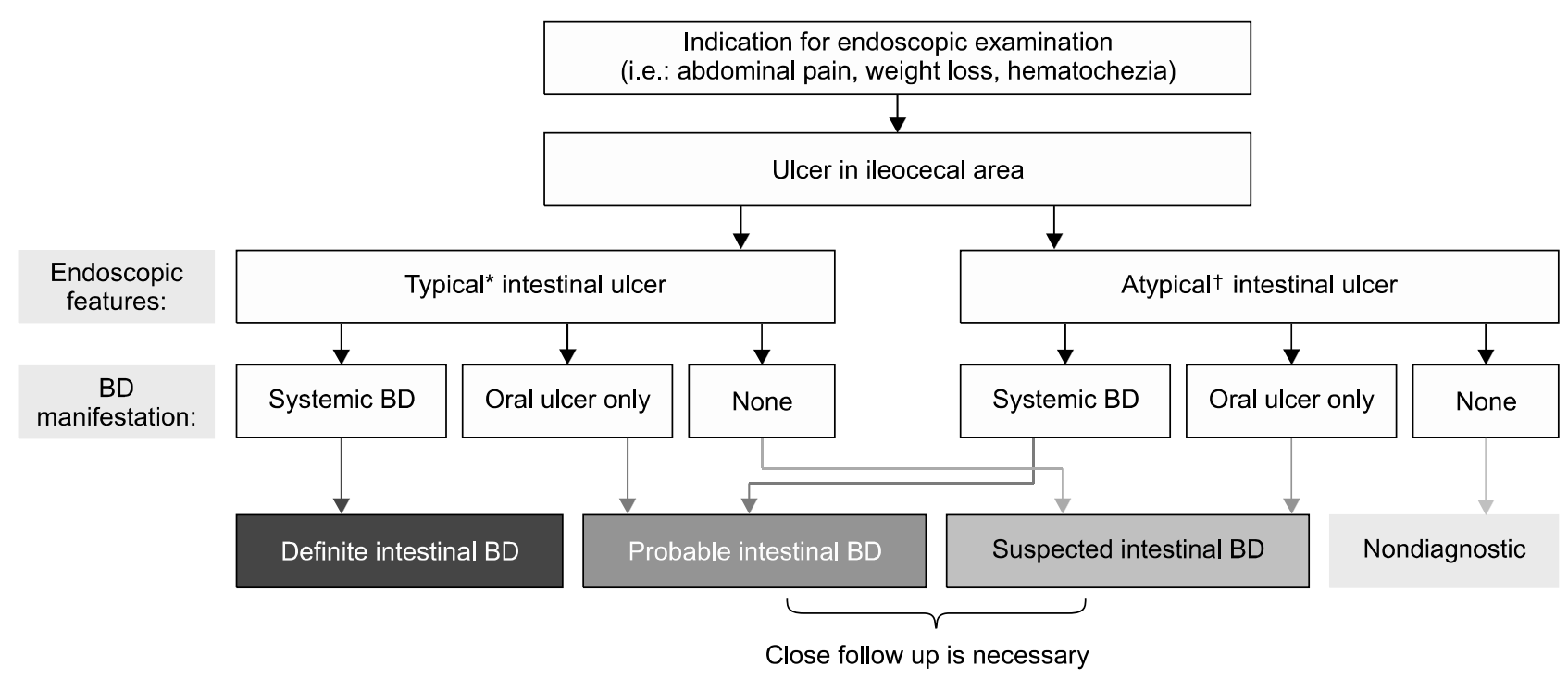

Figure 1. Diagnosis of intestinal Behçet's disease (BD) (adapted from the article of Cheon et al. Am J Gastroenterol 2009;104:2492-9 [10]). *Single or few deep oval-shaped ulcers with discrete margins in the ileocecal area, ${ }^{\dagger}$ aphthous, shallow ulcers. 
ation in the terminal ileum or inflammation in the small or large intestine is found along with clinical manifestations that satisfy the diagnostic criteria of $\mathrm{BD}$. These clinical findings are particularly useful for diagnosing patients whose symptoms do not fully meet the diagnostic criteria for systemic $\mathrm{BD}$ at the initial presentation. Basically, a diagnosis of intestinal $\mathrm{BD}$ relies on a combination of clinical, endoscopic, pathological, and radiological findings [11]. The clinical manifestations are similar to those of IBD, including diarrhea, hematochezia, weight loss, and abdominal pain with tenderness on the affected area [12]. The typical endoscopic features are oval-shaped large intestinal ulcerations in the ileocecal area with deep and discrete borders [8]. These features can vary from aphthous to deep penetrating volcano-shaped ulcerations and ulcerations with a more frequently focal to less frequently diffuse distribution [13]. The pathological findings in patients with intestinal BD are vasculitis involving the small and medium-sized vessels; lymphocyte infiltration in the perivascular space can also be detected $[14,15]$. Figure 1 presents the recommended diagnostic algorithm, which includes endoscopic observations and systemic BD criteria [10].

In diseases with unknown etiologies and no accurate diagnostic biomarkers, such as BD or IBD, the level of inflammation cannot be easily measured using a single parameter. Hence, a disease activity scoring system is needed to monitor the inflammatory status objectively and confirm the disease severity. The BD activity indices usually include the severity, location, and extent of the disease, and complications [16]. For intestinal BD, a simple and validated measurement tool called the Disease Activity Index of intestinal Behçet's disease (DAIBD) was proposed in 2011 by the Korean IBD Study Group for an assessment of the disease activity and establishing a therapeutic plan [17]. Before the development of the DAIBD, the Crohn's disease activity index (CDAI) had been used for intestinal BD owing to the similarities between the two diseases [3]. The DAIBD involves eight categories: general condition, extraintestinal manifestations, fever, abdominal pain, abdominal mass, abdominal tenderness, intestinal complications, and frequent liquid stools. The items are rated, with total scores ranging from 0 to 325 . The disease is categorized as quiescent, mild, moderate, and severe disease based on the total score $(\leq 19,20 \sim 39$, $40 \sim 74$, and $\geq 75$, respectively). The DAIBD is highly weighted on the general well-being and abdominal pain rather than on the other indices. Although the DAIBD is not correlated significantly with the endoscopic severity [18], it is more responsive and shows a better correlation with the physician's global assessment score than CDAI. Further studies will be needed in various ethnicities and for the validation of DAIBD regarding whether it can predict the disease course precisely based on endoscopic examinations and clinical symptoms.

\section{1) Recent findings of genetic and immune responses in intestinal BD}

Insights into the roles of genetic and immune responses in disease development or prognosis along with the development of molecular biological research techniques, including the genome-wide association studies (GWASs) and next-genome sequencing (NGS), have changed medical practice significantly [19]. The clinical symptoms and genetic features between intestinal $\mathrm{BD}$ and $\mathrm{BD}$ or IBD overlap; some genetic features of intestinal $\mathrm{BD}$ overlap with IBD, while others overlap with $\mathrm{BD}$. A human leukocyte antigen (HLA)-B51 allele and MHC class I-related gene A (MICA) are well-known genetic factors of BD [20-24], which have not been identified in IBD. In contrast, the recently identified factors, namely interleukin (IL) 10 and the IL23R-IL12RB2 loci, are associated with BD and IBD $[21,25]$. The recent findings from Korean patients with intestinal BD suggest that IL17A, IL23R, and STAT4 SNPs modulate the susceptibility to intestinal $\mathrm{BD}$. In particular, the haplotype of IL17A was observed to be a risk factor for intestinal BD development, whereas that of IL23R was associated with disease protection [26]. GWASs on intestinal BD first elucidated the genetic polymorphisms that contribute to disease development. They showed that NAALADL2 and YIPF7 have strong associations with the intestinal inflammation risk, thereby allowing the differentiation of intestinal BD from BD without intestinal involvement.

Regarding the contribution of the immunological response to triggering inflammation, similar to those in other autoimmune diseases, increased levels of Th1, Th17, $\mathrm{CD} 4^{+}$, and $\mathrm{CD} 8^{+} \mathrm{T}$ cell, and $\gamma \delta^{+} \mathrm{T}$ cell activities, as well as IL-12 and tumor necrosis factor alpha (TNF- $\alpha$ ) levels, were elevated in patients with BD [27-32]. Most of the proinflammatory cytokines associated with the innate immune system are activated in Crohn's disease and ulcerative colitis. This finding also applies to intestinal BD and has therapeutic implications. In patients with IBD, increasing numbers or activation of innate immune cells, including neutrophils, macrophages, and natural killer $\mathrm{T}$ 
cells, and adaptive immune cells, such as B and T cells, increase in the levels of TNF- $\alpha$, IL- $1 \beta$, interferon (IFN)- $\gamma$, and cytokines in the IL-23-Th17 pathway [33]. As with Crohn's disease, Th1- and Th17-related cytokines (IL-12, IL-23, and IL-27) are also upregulated in BD [34]. A recent study reported that the IL-12B levels correlated with the clinical and endoscopic disease activities of intestinal BD and IBD [35]. Proteomic analysis showed that the IL-1 $\beta$ and serum amyloid A levels were markedly higher in patients with intestinal BD [36]. These explanations of the pathophysiology of intestinal $\mathrm{BD}$ and the genet$\mathrm{ic} /$ clinical overlap between intestinal BD and IBD lead to a wide range of changes in the therapeutic environment of intestinal BD.

\section{2) Principles of the therapeutic approach}

Owing to the rarity of intestinal BD, scrutinized or systematic prospective clinical trials have not been prevalently conducted. Empirical and evidence-based therapeutic approaches have been established based on several retrospective studies and the similar pathophysiological background between intestinal BD and Crohn's disease, but the choice of treatment is still dependent on the physicians' opinion [4]. In this regard, Japanese researchers published consensus statements for the diagnosis and standard therapy for intestinal BD in 2007 [3]. Revised versions of the statements were published in 2014 [37] and 2020 [38], including the statement that an anti-TNF inhibitor can be considered standard therapy. The treatment algorithm, according to the disease severity, has been proposed based on the accumulated studies and experts' opinions in 2013 [39]. Considering the latest clinical trials and published data, this paper suggests an up-to-date treatment algorithm as follows (Figure 2). Basically, it is similar to the treatment procedure in the step-up therapy for IBD according to the disease severity (mild to moderate or severe) aiming to achieve and maintain clinical remission [40]. This is a sequential treatment procedure that is usually initiated by generally tolerable medications, such as 5-aminosalicylates, with escalation to the more effective but potentially more toxic drugs, including immunomodulators and biologics, for patients in whom each previous step of therapy has failed. With this algorithm, patients can avoid overtreatment and the unnecessary risk of adverse events, particularly in cases of a tolerable standard

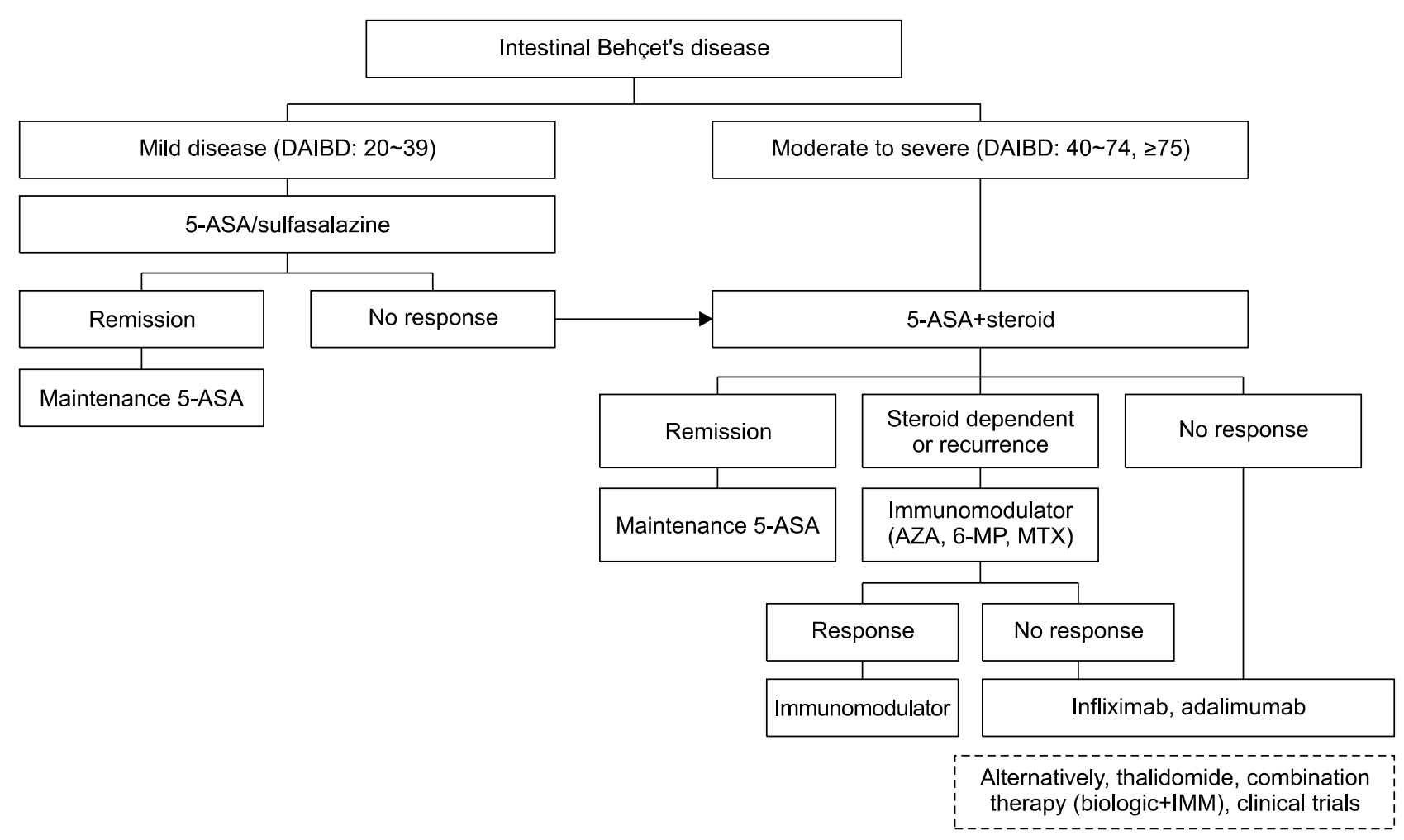

Figure 2. Treatment algorithm based on the disease severity (adapted from the article of Lee et al. Intest Res 2013;11:155-60 [39]). DAIBD: Disease Activity Index of intestinal Behçet's disease, 5-ASA: 5-Aminosalicylic acids, AZA: azathioprine, 6-MP: 6-mercaptopurine, MTX: methotrexate, IMM: immunomodulator. 
therapy. In IBD treatment, the top-down therapy strategy was proposed to induce rapid clinical remission with highly effective medications, such as early biologics, but no such attempts or research studies have been performed for intestinal BD. Surgery should be considered in a timely manner when the patient is suspected of severe complications or does not respond properly to the medical treatment. Complications, even irreversible ones, are likely to occur in accordance with a delayed decision of surgical intervention. Hence, the close discussion between gastroenterologists and surgeons is required. Therefore, a multidisciplinary approach should be available whenever their expertise is demanded in the intestinal BD treatment.

\section{3) 5-Aminosalicylic acids}

5-Aminosalicylic acids (5-ASA) have a similar mechanism to aspirin or non-steroidal anti-inflammatory drugs, which target the COX-2-inducing immunomodulatory actions to reduce intestinal inflammation [41]. 5-ASA inhibits COX-2 expression resulting from TNF- $\alpha /$ IL- $1 \beta$ [42]. The 5-ASAs used widely for IBD treatment, especially for patients with ulcerative colitis, are mesalamine, mesalazine, or sulfasalazine [43]. The clinical dosages of 5-ASA in patients with intestinal BD usually range from 2 to $4 \mathrm{~g}$ /day, while sulfasalazine is generally administered at 3 to $4 \mathrm{~g} /$ day [4]. Several clinical studies have obtained inconsistent results regarding the effectiveness of 5-ASA for intestinal BD. A case series study that included patients with intestinal BD reported that $79 \%$ patients showed symptomatic or colonoscopic improvement with sulfasalazine therapy [44]. A large-scale retrospective study conducted in 2012 showed that 5-ASA/sulfasalazine monotherapy was effective in maintaining remission, specifically with cumulative relapse rates of $46.7 \%$ at 10 years after remission. Younger age, disease severity, and higher C-reactive protein (CRP) level were associated with a poor prognosis with 5-ASA/sulfasalazine therapy [45]. A recent study investigated the induction response rate of oral 5-ASA and showed response and remission rates of $61 \%$ and $57 \%$, respectively, at week 8 [46]. Thus, 5-ASA can be recommended as induction and maintenance therapies for relatively mild to moderate intestinal BD. Conclusively, 5-ASA is a substantial therapeutic option that needs to be used in almost all patients with intestinal BD. On the other hand, patients with small bowel involvement are not indicated for sulfasalazine use.

\section{4) Corticosteroids}

Systemic corticosteroids are used effectively to control flare-ups in patients with moderate to severe BD and IBD in cases of failure of 5-ASA/sulfasalazine treatment [47-49]. Corticosteroids, which are well-known fast-acting anti-inflammatory drugs, are used widely with tapering strategies at a starting dosage of $0.5 \sim 1.0 \mathrm{mg} / \mathrm{kg} /$ day [3] that is tapered to $5 \mathrm{mg}$ each week and stopped within three months. Well-designed prospective clinical trials on the effectiveness of corticosteroids in patients with intestinal BD have not been conducted thus far. Some retrospective studies have suggested that corticosteroids are effective in the induction phase of intestinal BD. One retrospective cohort study [50] in Korea that included 54 patients reported that $46.3 \%(n=25)$ of patients with active intestinal $\mathrm{BD}$ achieved complete remission after one month of treatment, while $42.6 \%$ showed partial remission. After three months of treatment, $40.7 \%$ of the patients still responded to the corticosteroid treatment. One year after the initiation of corticosteroid administration, $48.1 \%$ of the patients remained responsive to the treatment, and $8.1 \%$ showed a prolonged response, but $35.2 \%$ had corticosteroid dependency, and $7.4 \%$ underwent intestinal surgery. Thus, it is recommended for inducing a short-term response in the early phase of the disease course, especially with severe systemic symptoms or severe disease activity. Considering that patients with intestinal BD with high-risk factors, such as gastrointestinal bleeding, mainly use this medication, side effects are the main burden for both physicians and patients because they increase the risk of gastrointestinal bleeding and perforation [51]. In addition, corticosteroid therapy was strongly associated with rebleeding in patients with intestinal BD (hazard ratio [HR], 3.2; 95\% confidence interval, 1.070 9.462; $\mathrm{p}=0.037$ ) [52]. Therefore, a tapering dose strategy should be maintained, and the prolonged use of prednisolone must not exceed $10 \mathrm{mg}$ /day. Systemic corticosteroids are often used too easily without hesitation, even if there are no symptoms. Moreover, they are often prolonged in clinical practice. Steroid usage should be based rigorously on the overall symptoms, and should not be used unconditionally due only to the presence of intestinal ulcers.

\section{5) Immunomodulators}

(1) Thiopurines

Thiopurine analogs, consisting of 6-mercaptopurine (6-MP) and its derivate, azathioprine (AZA), are func- 
tioning antimetabolites of nucleic acids that were introduced to inhibit the growth of rapidly dividing cells in the 1950s [53]. Thiopurines require an extensive metabolism to form thioguanine nucleotides (TGNs) because they are prodrugs that act specifically via the following three pathways: (1) inactivation of 6-MP to thiouric acid by xanthine oxidase and aldehyde oxidase, (2) 6-methyl MP (6-MMP) production by thiopurine methyltransferase (TPMT), (3) conversion of 6-MP to 6-TGN by enzymes, such as TPMT, inosine monophosphate dehydrogenase, and hypoxanthine phosphoribosyl transferase [54,55]. Regarding the mechanism of action of thiopurines, TPMT plays a major role in producing inactive 6-MMP and active 6-TGN by generating 6-methyl MP ribonucleotide (6-MMPR), and can ultimately increase 6-TGN production by converting 6-thioinosine monophosphate to 6-MMR indirectly [56]. The anti-inflammatory action of thiopurine is achieved by 6-TGN. In particular, it is integrated into leukocyte DNA and prevents DNA synthesis, which inhibits downstream T-cell proliferation and immunosuppression [57]. Based on this metabolism, AZA/6-MP is commonly indicated for patients with moderate to severe intestinal $\mathrm{BD}$, specifically those who are refractory or dependent on corticosteroids or show a secondary loss of response to anti-TNF- $\alpha$ agents. The drug is also used to reduce the postoperative recurrence rate after intestinal surgery [58].

AZA ( 25 to $50 \mathrm{mg} /$ day) is effective for both the cellular and humoral immune responses, particularly for the initial dose indication, in patients with intestinal $\mathrm{BD}$. The dose can be increased gradually every 2 to 3 weeks up to 2.0 to $2.5 \mathrm{mg} / \mathrm{kg}$, unless adverse drug reactions occur, such as leukopenia [59]. Although only one retrospective cohort study was conducted in patients with intestinal BD treated with AZA, it established the benefit of AZA as maintenance therapy. Jung et al. [59] evaluated 272 patients with intestinal BD who received thiopurine monotherapy and reported that $58.2 \%$ (39/67) of patients who received the first course of thiopurine remained in remission with thiopurine maintenance therapy. In this study, the cumulative relapse rates at 1, 2, 3, and 5 years after remission were $5.8 \%, 28.7 \%, 43.7 \%$, and $51.7 \%$, respectively. Younger age ( $<25$ years) at diagnosis and lower hemoglobin level $(<11 \mathrm{~g} / \mathrm{dL})$ were associated with a poor prognosis.

Similarly, Lee at al. [58] reviewed the postoperative recurrence rate of intestinal $\mathrm{BD}$ in patients who received thiopurine treatment, and the cumulative rate of clinical recurrence after surgery was significantly lower in the thiopurine group than in the 5-ASA treatment group $(\mathrm{p}=$ 0.050). Moreover, the HR of postoperative thiopurines compared to 5-ASA was 0.636 (range, 0.130 1.016; $\mathrm{p}=0.053$ ) for postoperative recurrence. AZA is an effective treatment for intestinal inflammation and can be used stably in the long term unless side effects occur. The most common and serious side effect is leukopenia, which increases the infection risk and even mortality [60]. Thus, the American Gastroenterological Association recommends that patients treated with AZA should be monitored routinely with complete blood counts [61].

With regard to IBD treatment, a genotype-based strategy for thiopurine treatment using the TPMT, FTO, and NUDT15 variants to prevent and reduce myelosuppression was investigated [62]. Including intestinal BD patients, that prospective study assigned seventy-two patients randomly to a group with genotype analysis (NUDT15 variant, FTO variant, TPMT variants) and a non-genotyping group. Myelosuppression was more frequent in the non-genotyping group than the pretreatment genotype analysis group $(35.9 \%, 16.7 \%$, respectively, $\mathrm{p}=0.005)$. Recognizing genetic information in advance and adjusting the treatment plans also led to a decrease in the numbers and frequencies of outpatient clinic visits, drug discontinuation, and dose reductions [62]. Azathioprine is a beneficial therapeutic option, but it is still difficult to use for patients with hematological disorders, such as aplastic anemia and myelodysplastic syndrome (MDS), because of the potential side effects. Further prospective randomized placebo-controlled trials with more patients will be necessary for elucidating a safer thiopurine treatment for patients with intestinal BD.

(2) Methotrexate

Methotrexate (MTX) is an analog of folic acid and aminopterin that was first manufactured in 1945 and is a long-standing medication that has been used for patients with cancer since the 1950s [63]. Recently, it has gained renewed attention as a notable therapy for autoimmune diseases. Low-dose MTX $(25 \mathrm{mg} /$ day for the induction phase) appears to be effective in the treatment of rheumatoid arthritis, neuro-BD, uveitis, and other types of $\mathrm{BD}$ involvement [64-66]. In addition, MTX is used increasingly in combination with biologics owing to the immunogenicity of anti-TNF inhibitors [67]. The mechanism of immunosuppression by MTX mainly involves the reduction of cell proliferation, an increase in the rate of apoptosis of T-cells, and endogenous adenosine accumulation. 
Hence, it can alter cytokine production, humoral responses, and bone formation, which leads to the deactivation of the immune system [68]. The effectiveness and safety of MTX for intestinal BD have rarely been investigated. A case series of 10 patients with refractory intestinal BD reported improved symptoms and disease-related complications within four weeks of MTX treatment in 2011 [69]. Moreover, the ulcers disappeared in nine of the patients at 12 months.

Park et al. [70] published retrospective data on the efficacy of MTX therapy, specifically MTX monotherapy and combination therapy with MTX and adalimumab (ADA), for intestinal BD in 2018. Among 10 patients, three and five attained steroid-free remission at three and six months, respectively. The serum CRP level, erythrocyte sedimentation rate, and DAIBD score decreased at six months compared to their baseline values, but only the decrease in CRP levels was statistically significant $(\mathrm{p}=$ 0.039) [70].

(3) Other immunomodulators

Immunomodulators, including cyclosporine, tacrolimus, interferon (IFN), and intravenous immunoglobulin (IVIG), are considered alternative therapeutic options for intestinal BD based on their pharmaceutical mechanism. On the other hand, no sufficient scientific evidence exists because of the relatively few cases reported, and no prospective clinical trials of immunomodulators have been conducted in patients with intestinal BD. Cyclosporine, which decreases the levels of inflammatory cytokines in T-lymphocytes by blocking the phosphatase activity of calcineurin [71], is commonly used for autoimmune diseases, such as rheumatoid arthritis, psoriasis, and ocular manifestations of BD [72,73]. According to the updated European League Against Rheumatism guideline, nervous system involvement is not indicated for cyclosporine based on level 3 evidence [74]. Bayraktar et al. [75] and the unpublished experience from Severance Hospital suggest that cyclosporine therapy has no clinical benefit for patients with intestinal BD. Tacrolimus, a macrocyclic lactone with potent immunosuppressive properties [76], is a standard immunosuppressive regimen after renal transplantation [77]. One case was reported, and clinical improvement was observed in patients with intestinal BD refractory to conventional therapies [78]. IFN and IVIG were introduced in the 1950s and have been used for arthritis, $\mathrm{BD}[79,80]$, and other autoimmune diseases.

On the other hand, studies examining the role of these therapies in intestinal $\mathrm{BD}$ are limited. Therefore, the effi- cacy of IFN or IVIG for intestinal BD is unclear. A study of patients with $B D$ who were unresponsive to systemic steroids and/or immunosuppressants was conducted using subcutaneous IFN- $\alpha$ at a dosage of $6 \times 10^{6} \mathrm{IU} /$ day three times per week for two months. In that study, $75 \%(9 / 12)$ of the patients achieved complete remission, $16.6 \%$ $(2 / 12)$ had partial remission, and $8.3 \%$ showed no response [81]. In 1998, a 32-year-old Caucasian woman with intestinal BD was treated with IVIG. Her symptoms improved after IVIG treatment initiation, and the intestinal lesions disappeared after six weeks of IVIG therapy at $400 \mathrm{mg} / \mathrm{kg} /$ day for five days [82]. Nevertheless, further evidence and clinical trials will be needed to prove their efficacy.

\section{6) Thalidomide}

Thalidomide was first used as a sedative in the 1950s and withdrawn from the European pharmaceutical market in the 1960s owing to its teratogenic effects in early pregnancy, including severe birth defects, such as phocomelia [83]. This was defined as a "thalidomide tragedy" and has been a major issue in the history of biomedical ethics and drug safety monitoring. Eventually, it contributed to the approval system and toxicology guidelines of the United States Food and Drug Administration $[84,85]$. After several clinical trials over a decade, thalidomide has now been approved and used for the treatment of inflammatory diseases, including leprosy, rheumatoid arthritis, $\mathrm{BD}$, and even multiple myeloma or malignant B-cell lymphoma [86-88]. Thalidomide, a synthetic derivative of glutamic acid, was suggested to be effective against the mucocutaneous and follicular lesions of BD based on the results of a randomized, double-blind, placebo-controlled study [89]. A case series of BD with intestinal involvement reported that thalidomide was effective for recurrent perforating intestinal ulcers and led to clinical improvement in terms of corticosteroid-free remission [90,91].

These outcomes were observed consistently in another case series in Korea [92]. Patients with recurrent intestinal BD refractory to conventional treatments, including 5-ASA and immunomodulators, reported a response to thalidomide with clinical and radiological improvement. The major side effects observed were edema, neutropenia, and sepsis. Similarly, a prospective open-label study of patients with IBD treated with thalidomide reported clinical response rates of $83.3 \%$ and $100.0 \%$ for Crohn's disease and ulcerative colitis at week 12 [93]. 
Definitive dose guidelines for intestinal $\mathrm{BD}$ are not available. On the other hand, an initial thalidomide dosage of $2 \mathrm{mg} / \mathrm{kg} /$ day has been used, and dose adjustment according to the treatment response is needed [90]. In addition, thalidomide appears to exert a selective effect on TNF- $\alpha$ production by the degradation of its encoding messenger RNA [94]. Thus, the efficacy and safety of anti-TNF inhibitor or thalidomide therapy were investigated in patients with intestinal BD who were refractory to conventional therapies [95]. Of 13 patients, 10 (75\%) achieved clinical and endoscopic remission with TNF- $\alpha$ antagonists and/or thalidomide therapy. This result also supports the efficacy of thalidomide as a therapeutic option for intestinal BD. Nevertheless, well-designed prospective trials are still needed, and rigorous monitoring of the side effects is warranted.

\section{7) Biologics}

While recent advances in the novel therapeutic armamentarium, including biologics for autoimmune diseases, have made transformative changes in therapeutic environments. Several biologic agents are now available for patients with autoimmune diseases, particularly those who do not respond to conventional therapies or those who develop serious adverse events. The use of these biologics has also been attempted in patients with intestinal $\mathrm{BD}$. As mentioned previously, the pathogenesis of intestinal $\mathrm{BD}$ is related to an abnormal T-cell immune response and cytokines derived from T helper type 1 (Th1) lymphocytes, including TNF- $\alpha$, IFN- $\gamma$, IL-12, and IL-18 $[96,97]$. The TNF- $\alpha$ expression level in the blood increases, mucosal damage occurs, and clinical symptoms worsen [98]. Therefore, biologics targeting TNF- $\alpha$ have been administered to patients with intestinal BD with these scientific backgrounds. Currently, anti-TNF- $\alpha$ monoclonal antibodies, including infliximab (IFX) and $\mathrm{ADA}$, are considered standard therapies for patients with intestinal $\mathrm{BD}$ [37].

(1) Infliximab

IFX, a TNF blocker, is indicated for patients with IBD, arthritis, ankylosing spondylitis, and psoriasis, and who have shown an inadequate response to conventional therapy [4]. A dose of $5 \mathrm{mg} / \mathrm{kg}$ at zero, two, and six weeks is suggested for induction therapy and then every eight weeks for maintenance therapy. Some adult patients who respond initially to treatment may benefit from increasing the dose to $10 \mathrm{mg} / \mathrm{kg}$ if they later become unresponsive to this treatment [37]. The first case report of
IFX use for intestinal BD was in 2001, which showed a decreased CDAI score from 270 to 13 points by week two, and endoscopic and histological improvements at week 10 [99]. The following case reports consistently suggested the positive efficacy of IFX for intestinal BD as both induction and maintenance therapy $[100,101]$. A multicenter retrospective study in patients with moderate to severe intestinal BD reported the results of IFX treatment from 28 patients in Korean tertiary hospitals [102]. The clinical response rates at 2, 4, 30, and 54 weeks were $75 \%, 64.3 \%, 50 \%$, and $39.1 \%$, respectively, and the clinical remission rate at week 30 was $46.2 \%$. During the follow-up period, one case of serious infection was observed, but no malignancies or deaths occurred. The efficacy and safety of IFX were investigated in a prospective open-label phase 3 study in 2016, which included 18 patients with $\mathrm{BD}$. Among the patients enrolled, 11 had intestinal involvement and were refractory to conventional therapies [103]. The IFX dose was the same as that for Crohn's disease and ulcerative colitis. A complete response was observed in $61 \%(11 / 18)$ of patients at weeks 14 and 30, and the patients remained in remission until week 54. The CRP levels were decreased after week two, and most of the subjects showed healed ulcers at week 14 . Despite these results, as with patients with IBD, the patients with intestinal BD in this study also showed a primary and secondary loss of response. Hence, the need to increase the dose at week 30 or treatment failure were observed [103]. An interventional, open-label, single-arm, multicenter study of IFX in 33 patients with moderate to severe refractory intestinal BD is ongoing in Korea (ClinicalTrials.gov NCT02505568). This study is expected to provide more scientific evidence for the effectiveness of IFX treatment for patients with intestinal BD. Studies are still lacking, but evidence for the positive efficacy of IFX combination therapy with immunomodulators has been reported. In a previous study [69], all 10 patients showed improvement in the intestinal symptoms and complications within four weeks. The rate of disappearance of ileocecal ulcerations was 50\% (5/10) and $90 \%(9 / 10)$ at 6 and 12 months, respectively [69]. Combination therapy of biologics with immunomodulators is beneficial for reducing the immunogenicity and increasing the serum levels, but the risks of infection and malignancy need to be considered carefully [104].

(2) Adalimumab

ADA is a fully human anti-TNF- $\alpha$ monoclonal antibody, whereas IFX is a chimeric monoclonal antibody against 
TNF- $\alpha$. The doses for patients with intestinal BD can be used for induction therapy at doses of 160,80 , and $40 \mathrm{mg}$ at week zero (baseline), two, and four subcutaneously, and then $40 \mathrm{mg}$ every other week for responders [105]. De Cassan et al. [106] described their experience with ADA use as the first anti-TNF- $\alpha$ inhibitor in two patients with familial intestinal $\mathrm{BD}$, who were steroid dependent. Rapid clinical remission was observed in the induction phase, which was sustained in the maintenance phase.

The following case report suggested the efficacy of ADA for intestinal BD with myelodysplastic syndrome based on successful improvement in gastrointestinal symptoms, CRP levels, leukocytopenia, and anemia four months after the ADA treatment [107]. After that, a prospective multicenter open-label study was conducted in Japan that involved 20 patients with intestinal BD, who were refractory to conventional therapies, such as corticosteroids or immunomodulators [105]. Nine patients (45\%) achieved the primary endpoints after 24 weeks, which were an alleviation of gastrointestinal symptoms and a decrease in the endoscopic assessment score to 1 or lower than the baseline score. In addition, ADA treatment induced complete remission in $20 \%$ of patients at week 24 , which was maintained until week 52 , but no notable safety issues were observed. A recent phase 3 study in Japan evaluated the efficacy and long-term safety profiles of ADA treatment in 20 patients with intestinal BD. Fifteen of the patients remained in the study until 100 weeks of follow-up. Significant improvement, which was defined based on the gastrointestinal symptom and endoscopic scores, was observed in $60 \%$ of patients at week 52 and $40 \%$ at week 100 . Of the patients, $20 \%$ and $15 \%$ showed clinical remission at weeks 52 and 100, respectively, with tolerable safety profiles [108]. These results played a pivotal role in gaining approval of the use of ADA for intestinal BD treatment in Japan. Combination therapy using ADA with other immunomodulatory medications has not yet been actively investigated. Vitale et al., however, compared the efficacy and safety of ADA monotherapy and combination therapy with disease-modifying antirheumatic drugs. In this study, 100 patients with BD were enrolled; no significant difference in clinical outcomes was observed between the two groups [109].

A study in 2019 examined the effectiveness of anti-TNFs in patients with intestinal BD compared to that of corticosteroids without anti-TNF. Both groups showed improvement in the DAIBD score one year after treatment (85.2 \pm 29.6 to $40.5 \pm 44.7$ in the corticosteroid group and
$64.7 \pm 34.9$ to $21.1 \pm 28.9$ in the anti-TNF group). Anti-TNF administration was effective in reducing the concomitant steroid dose to $<7.5 \mathrm{mg}(\mathrm{p}=0.0001)$. Considering that one patient discontinued treatment because of bacterial infection, the potential risk of intestinal perforation or massive bleeding should be considered when using steroids [110]. A recently published study that used real-world data on ADA and IFX for patients with refractory intestinal $\mathrm{BD}$ suggested switching from an anti-TNF inhibitor to another if a patient is refractory to the first-line anti-TNF inhibitor [111]. Biologics can be ineffective in cases of urgent patients who require surgical intervention. Therefore, a decision should be made under a multi-disciplinary discussion, including gastroenterologists, rheumatologists, and surgeons, as to whether to discontinue the treatment and perform surgery.

\section{CONCLUSION}

The prevalence of intestinal BD is low in Western countries but relatively high in East Asian countries, including Korea. Thus, there are limited clinical trial data with intestinal BD due to the general lack of disease awareness, small number of cases, and difficulty in subject recruitment. In addition, it is a relapsing, complex systemic inflammatory disease that is often unpredictable and has a "wax and wane" disease course with massive bleeding and perforation. Patients are easily refractory to conventional therapies, which caused new biologics to evolve in the treatment of intestinal BD. Intestinal BD can be treated using the same therapeutic approaches for $\mathrm{BD}$, but the differences in intestinal $\mathrm{BD}$ from other types of $\mathrm{BD}$ in terms of the intestinal specific pathophysiology and treatment response must be considered. Thus far, the IBD treatment guidelines are effective in the treatment of intestinal BD. Several retrospective studies or clinical trials with a small number of patients have been the mainstay for gathering evidence on intestinal BD treatment. On the other hand, recent prospective and scientifically designed clinical trials specifically for intestinal BD have actively been conducted. Therefore, an effective treatment guideline is expected to be established soon. As for biologics, many novel agents with different targets have emerged for autoimmune diseases, including IL 12/23, Janus kinases, and IL-6 inhibitors. Further clinical research focusing on intestinal $\mathrm{BD}$ will be needed to obtain scientific evidence and provide treatment opportunities to the patients. Ultimately, close cooperation between rheuma- 
tology, gastroenterology, and colorectal surgery departments as multidisciplinary care is essential for the treatment of intestinal BD.

\section{ACKNOWLEDGMENTS}

The author wishes to thank Sinyoung Park, who assisted in collecting and analyzing the related literature, and writing and revising the manuscript.

\section{CONFLICT OF INTEREST}

No potential conflict of interest relevant to this article was reported.

\section{REFERENCES}

1. Dilsen N. History and development of Behçet's disease. Rev Rhum Engl Ed 1996;63:512-9.

2. Nakata K, Murakami T, Hashi N, Tsutsumi S. Neuro-Behçet's syndrome. Report of an autopsy case. Bull Osaka Med Sch 1964;10:105-19.

3. Kobayashi K, Ueno F, Bito S, Iwao Y, Fukushima T, Hiwatashi N, et al. Development of consensus statements for the diagnosis and management of intestinal Behçet's disease using a modified Delphi approach. J Gastroeenterol 2007;42:737-45.

4. Cheon JH, Kim WH. An update on the diagnosis, treatment, and prognosis of intestinal Behçet's disease. Curr Opin Rheumatol 2015;27:24-31.

5. Han M, Jung YS, Kim WH, Cheon JH, Park S. Incidence and clinical outcomes of intestinal Behçet's disease in Korea, 2011-2014: a nationwide population-based study. J Gastroenterol 2017;52:920-8.

6. Mizushima Y. Recent research into Behçet's disease in Japan. Int J Tissue React 1988;10:59-65.

7. International Study Group for Behçet's Disease. Criteria for diagnosis of Behçet's disease. Lancet 1990;335: 1078-80.

8. Lee CR, Kim WH, Cho YS, Kim MH, Kim JH, Park IS, et al. Colonoscopic findings in intestinal Behçet's disease. Inflamm Bowel Dis 2001;7:243-9.

9. Jung HC, Rhee PL, Song IS, Choi KW, Kim CY. Temporal changes in the clinical type or diagnosis of Behçet's colitis in patients with aphthoid or punched-out colonic ulcerations. J Korean Med Sci 1991;6:313-8.

10. Cheon JH, Kim ES, Shin SJ, Kim TI, Lee KM, Kim SW, et al. Development and validation of novel diagnostic criteria for intestinal Behçet's disease in Korean patients with ileocolonic ulcers. Am J Gastroenterol 2009;104:2492-9.

11. Cheon JH, Shin SJ, Kim SW, Lee KM, Kim JS, Kim WH; IBD Study Group of the Korean Association of the Study of Intestinal Diseases. Diagnosis of intestinal Behçet's disease. Korean J Gastroenterol 2009;53:187-93.

12. Choi IJ, Kim JS, Cha SD, Jung HC, Park JG, Song IS, et al. Long-term clinical course and prognostic factors in in- testinal Behçet's disease. Dis Colon Rectum 2000;43: 692-700.

13. Kim JS, Lim SH, Choi IJ, Moon H, Jung HC, Song IS, et al. Prediction of the clinical course of Behçet's colitis according to macroscopic classification by colonoscopy. Endoscopy 2000;32:635-40.

14. Shepherd NA. Pathological mimics of chronic inflammatory bowel disease. J Clin Pathol 1991;44:726-33.

15. Ebert EC. Gastrointestinal manifestations of Behçet's disease. Dig Dis Sci 2009;54:201-7.

16. Lawton G, Bhakta BB, Chamberlain MA, Tennant A. The Behcet's disease activity index. Rheumatology (Oxford) 2004;43:73-8.

17. Cheon JH, Han DS, Park JY, Ye BD, Jung SA, Park YS, et al.; Korean IBD Study Group. Development, validation, and responsiveness of a novel disease activity index for intestinal Behçet's disease. Inflamm Bowel Dis 2011;17: 605-13.

18. Lee HJ, Kim YN, Jang HW, Jeon HH, Jung ES, Park SJ, et al. Correlations between endoscopic and clinical disease activity indices in intestinal Behcet's disease. World J Gastroenterol 2012;18:5771-8.

19. Medzhitov R, Shevach EM, Trinchieri G, Mellor AL, Munn $\mathrm{DH}$, Gordon S, et al. Highlights of 10 years of immunology in Nature Reviews Immunology. Nat Rev Immunol 2011; 11:693-702.

20. Karasneh J, Gül A, Ollier WE, Silman AJ, Worthington J. Whole-genome screening for susceptibility genes in multicase families with Behçet's disease. Arthritis Rheum 2005; 52:1836-42.

21. Remmers EF, Cosan F, Kirino Y, Ombrello MJ, Abaci N, Satorius C, et al. Genome-wide association study identifies variants in the MHC class I, IL10, and IL23R-IL12RB2 regions associated with Behçet's disease. Nat Genet 2010; 42:698-702.

22. Mizuki N, Meguro A, Ota M, Ohno S, Shiota T, Kawagoe T, et al. Genome-wide association studies identify IL23RIL12RB2 and IL10 as Behçet's disease susceptibility loci. Nat Genet 2010;42:703-6.

23. Direskeneli H. Behçet's disease: infectious aetiology, new autoantigens, and HLA-B51. Ann Rheum Dis 2001;60: 996-1002.

24. Ohno S, Ohguchi M, Hirose S, Matsuda H, Wakisaka A, Aizawa M. Close association of HLA-Bw51 with Behçet's disease. Arch Ophthalmol 1982;100:1455-8.

25. Franke A, McGovern DP, Barrett JC, Wang K, Radford-Smith GL, Ahmad T, et al. Genome-wide meta-analysis increases to 71 the number of confirmed Crohn's disease susceptibility loci. Nat Genet 2010;42:1118-25.

26. Kim ES, Kim SW, Moon CM, Park JJ, Kim TI, Kim WH, et al. Interactions between IL17A, IL23R, and STAT4 polymorphisms confer susceptibility to intestinal Behcet's disease in Korean population. Life Sci 2012;90:740-6.

27. Sayinalp N, Ozcebe OI, Ozdemir O, Haznedaroğlu IC, Dündar S, Kirazli S. Cytokines in Behçet's disease. J Rheumatol 1996;23:321-2.

28. Suzuki Y, Hoshi K, Matsuda T, Mizushima Y. Increased peripheral blood gamma delta $+\mathrm{T}$ cells and natural killer cells in Behçet's disease. J Rheumatol 1992;19:588-92.

29. Sugi-Ikai N, Nakazawa M, Nakamura S, Ohno S, Minami M. Increased frequencies of interleukin-2- and interfer- 
on-gamma-producing T cells in patients with active Behçet's disease. Invest Ophthalmol Vis Sci 1998;39:996-1004.

30. Direskeneli H, Eksioglu-Demiralp E, Kibaroglu A, Yavuz S, Ergun T, Akoglu T. Oligoclonal T cesl expansions in patients with Behçet's disease. Clin Exp Immunol 1999; 117:166-70.

31. Freysdottir J, Lau S, Fortune F. Gammadelta T cells in Behçet's disease (BD) and recurrent aphthous stomatitis (RAS). Clin Exp Immunol 1999;118:451-7.

32. Na SY, Park MJ, Park S, Lee ES. Up-regulation of Th17 and related cytokines in Behçet's disease corresponding to disease activity. Clin Exp Rheumatol 2013;31(3 Suppl 77): $32-40$.

33. Abraham $\mathrm{C}, \mathrm{Cho} \mathrm{JH}$. Inflammatory bowel disease. $\mathrm{N}$ Engl J Med 2009;361:2066-78.

34. Sartor RB. Mechanisms of disease: pathogenesis of Crohn's disease and ulcerative colitis. Nat Clin Pract Gastroenterol Hepatol 2006;3:390-407.

35. Lee HW, Chung SH, Moon CM, Che X, Kim SW, Park SJ, et al. The correlation of serum IL-12B expression with disease activity in patients with inflammatory bowel disease. Medicine (Baltimore) 2016;95:e3772.

36. Lee HJ, Kim JH, Kim SW, Joo HA, Lee HW, Kim YS, et al. Proteomic analysis of serum amyloid a as a potential marker in intestinal Behçet's disease. Dig Dis Sci 2017;62: 1953-62.

37. Hisamatsu T, Ueno F, Matsumoto T, Kobayashi K, Koganei K, Kunisaki R, et al. The 2 nd edition of consensus statements for the diagnosis and management of intestinal Behçet's disease: indication of anti-TNF $\alpha$ monoclonal antibodies. J Gastroenterol 2014;49:156-62.

38. Watanabe K, Tanida S, Inoue N, Kunisaki R, Kobayashi K, Nagahori M, et al. Evidence-based diagnosis and clinical practice guidelines for intestinal Behçet's disease 2020 edited by Intractable Diseases, the Health and Labour Sciences Research Grants. J Gastroenterol 2020;55: 679-700.

39. Lee HW, Kim WH, Cheon JH. The medical treatments of intestinal Behçet's disease: an update. Intest Res 2013;11:155-60.

40. Baert F, Caprilli R, Angelucci E. Medical therapy for Crohn's disease: top-down or step-up? Dig Dis 2007;25: 260-6.

41. Stolfi C, Pellegrini R, Franze E, Pallone F, Monteleone G. Molecular basis of the potential of mesalazine to prevent colorectal cancer. World J Gastroenterol 2008;14:4434-9.

42. Stolfi C, Fina D, Caruso R, Caprioli F, Sarra M, Fantini MC, et al. Cyclooxygenase-2-dependent and -independent inhibition of proliferation of colon cancer cells by 5 -aminosalicylic acid. Biochem Pharmacol 2008;75:668-76.

43. Choi CH, Moon W, Kim YS, Kim ES, Lee BI, Jung Y, et al.; IBD Study Group of the Korean Association for the Study of the Intestinal Diseases. Second Korean guideline for the management of ulcerative colitis. Korean J Gastroenterol 2017;69:1-28.

44. Yoo HM, Han KH, Kim PS, Kim WH, Kang JK, Park IS, et al. Clinical features of intestinal Behoet's disease and therapeutic effects of sulfasalazine. Korean J Gastroenterol 1997;29:465-72.

45. Jung YS, Hong SP, Kim TI, Kim WH, Cheon JH. Long-term clinical outcomes and factors predictive of relapse after 5-aminosalicylate or sulfasalazine therapy in patients with intestinal Behcet disease. J Clin Gastroenterol 2012;46: e38-45.

46. Kinoshita H, Nishioka H, Ikeda A, Ikoma K, Sameshima Y, $\mathrm{Ohi} \mathrm{H}$, et al. Remission induction, maintenance, and endoscopic outcome with oral 5-aminosalicylic acid in intestinal Behçet's disease. J Gastroenterol Hepatol 2019; 34:1929-39.

47. Karadag O, Bolek EC. Management of Behcet's syndrome. Rheumatology (Oxford) 2020;59(Supple 3):iii108-17.

48. Park JJ, Yang SK, Ye BD, Kim JW, Park DI, Yoon H, et al.; IBD Study Group of the Korean Association for the Study of Intestinal Diseases. Second Korean guidelines for the management of Crohn's disease. Intest Res 2017;15: 38-67.

49. Choi CH, Moon W, Kim YS, Kim ES, Lee BI, Jung Y, et al.; IBD Study Group of the Korean Association for the Study of Intestinal Diseases. Second Korean guidelines for the management of ulcerative colitis. Intest Res 2017;15:7-37.

50. Park JJ, Kim WH, Cheon JH. Outcome predictors for intestinal Behçet's disease. Yonsei Med J 2013;54:1084-90.

51. Narum S, Westergren T, Klemp M. Corticosteroids and risk of gastrointestinal bleeding: a systematic review and meta-analysis. BMJ Open 2014;4:e004587.

52. Park J, Cheon JH, Park YE, Lee YJ, Lee HJ, Park SJ, et al. Risk factors and outcomes of acute lower gastrointestinal bleeding in intestinal Behçet's disease. Int J Colorectal Dis 2017;32:745-51.

53. Elion GB. The purine path to chemotherapy. Science 1989; 244:41-7.

54. González-Lama Y, Gisbert JP. Monitoring thiopurine metabolites in inflammatory bowel disease. Frontline Gastroenterol 2016;7:301-7.

55. Dubinsky MC. Azathioprine, 6-mercaptopurine in inflammatory bowel disease: pharmacology, efficacy, and safety. Clin Gastroenterol Hepatol 2004;2:731-43.

56. Derijks LJ, Gilissen LP, Engels LG, Bos LP, Bus PJ, Lohman $\mathrm{JJ}$, et al. Pharmacokinetics of 6-thioguanine in patients with inflammatory bowel disease. Ther Drug Monit 2006; 28:45-50.

57. Chang JY, Cheon JH. Thiopurine therapy in patients with inflammatory bowel disease: a focus on metabolism and pharmacogenetics. Dig Dis Sci 2019;64:2395-403.

58. Lee HW, Cheon JH, Lee HJ, Park SJ, Hong SP, Kim TI, et al. Postoperative effects of thiopurines in patients with intestinal Behçet's disease. Dig Dis Sci 2015;60:3721-7.

59. Jung YS, Cheon JH, Hong SP, Kim TI, Kim WH. Clinical outcomes and prognostic factors for thiopurine maintenance therapy in patients with intestinal Behcet's disease. Inflamm Bowel Dis 2012;18:750-7.

60. Connell WR, Kamm MA, Ritchie JK, Lennard-Jones JE. Bone marrow toxicity caused by azathioprine in inflammatory bowel disease: 27 years of experience. Gut 1993;34:1081-5

61. Feuerstein JD, Nguyen GC, Kupfer SS, Falck-Ytter Y, Singh $\mathrm{S}$; American Gastroenterological Association Institute Clinical Guidelines Committee. American Gastroenterological Association Institute guideline on therapeutic drug monitoring in inflammatory bowel disease. Gastroenterology 2017;153:827-34.

62. Chang JY, Park SJ, Jung ES, Jung SA, Moon CM, Chun J, et 
al. Genotype-based treatment with thiopurine reduces incidence of myelosuppression in patients with inflammatory bowel diseases. Clin Gastroenterol Hepatol 2020;18:20108.e2.

63. Bannwarth B, Labat L, Moride Y, Schaeverbeke T. Methotrexate in rheumatoid arthritis. An update. Drugs 1994;47:25-50.

64. Malaviya AN. Does methotrexate cause interstitial lung disease in rheumatoid arthritis: what is the evidence? Int J Rheum Dis 2020;23:713-6.

65. Borhani-Haghighi A, Kardeh B, Banerjee S, Yadollahikhales G, Safari A, Sahraian MA, et al. Neuro-Behcet's disease: an update on diagnosis, differential diagnoses, and treatment. Mult Scler Relat Disord 2019;39:101906.

66. Khalil HE, El Gendy HA, Youssef HA, Haroun HE, Gheita TA, Bakir HM. The effectiveness of intraocular methotrexate in the treatment of posterior uveitis in Behçet's disease patients compared to retrobulbar steroids injection. J Ophthalmol 2016;2016:1678495.

67. Feagan BG, McDonald JW, Panaccione R, Enns RA, Bernstein CN, Ponich TP, et al. Methotrexate in combination with infliximab is no more effective than infliximab alone in patients with Crohn's disease. Gastroenterology 2014;146:681-8.e1.

68. Wessels JA, Huizinga TW, Guchelaar HJ. Recent insights in the pharmacological actions of methotrexate in the treatment of rheumatoid arthritis. Rheumatology (Oxford) 2008;47:249-55.

69. Iwata S, Saito K, Yamaoka K, Tsujimura S, Nawata M, Hanami K, et al. Efficacy of combination therapy of anti-TNF- $\alpha$ antibody infliximab and methotrexate in refractory entero-Behçet's disease. Mod Rheumatol 2011; 21:184-91.

70. Park J, Cheon JH, Park Y, Park SJ, Kim TI, Kim WH. Efficacy and tolerability of methotrexate therapy for refractory intestinal Behçet's disease: a single center experience. Intest Res 2018;16:315-8.

71. Matsuda S, Koyasu S. Mechanisms of action of cyclosporine. Immunopharmacology 2000;47:119-25.

72. Hatemi G, Silman A, Bang D, Bodaghi B, Chamberlain AM, Gul A, et al.; EULAR Expert Committee. EULAR recommendations for the management of Behçet disease. Ann Rheum Dis 2008;67:1656-62.

73. Ozdal PC, Ortaç S, Taskintuna I, Firat E. Long-term therapy with low dose cyclosporin A in ocular Behçet's disease. Doc Ophthalmol 2002;105:301-12.

74. Hatemi G, Christensen R, Bang D, Bodaghi B, Celik AF, Fortune F, et al. 2018 update of the EULAR recommendations for the management of Behçet's syndrome. Ann Rheum Dis 2018;77:808-18.

75. Bayraktar Y, Ozaslan E, Van Thiel DH. Gastrointestinal manifestations of Behcet's disease. J Clin Gastroenterol 2000;30:144-54.

76. Venkataramanan R, Swaminathan A, Prasad T, Jain A, Zuckerman S, Warty V, et al. Clinical pharmacokinetics of tacrolimus. Clin Pharmacokinet 1995;29:404-30.

77. Schutte-Nutgen K, Tholking G, Suwelack B, Reuter S. Tacrolimus - pharmacokinetic considerations for clinicians. Curr Drug Metab 2018;19:342-50.

78. Matsumura K, Nakase H, Chiba T. Efficacy of oral tacrolimus on intestinal Behcet's disease. Inflamm Bowel Dis
2010;16:188-9.

79. Cantarini L, Stromillo ML, Vitale A, Lopalco G, Emmi G, Silvestri E, et al. Efficacy and safety of intravenous immunoglobulin treatment in refractory Behcet's disease with different organ involvement: a case series. Isr Med Assoc J 2016;18:238-42.

80. Alpsoy E, Durusoy C, Yilmaz E, Ozgurel Y, Ermis O, Yazar $\mathrm{S}$, et al. Interferon alfa-2a in the treatment of Behçet disease: a randomized placebo-controlled and double-blind study. Arch Dermatol 2002;138:467-71.

81. Georgiou S, Monastirli A, Pasmatzi E, Gartaganis S, Goerz G, Tsambaos D. Efficacy and safety of systemic recombinant interferon-alpha in Behçet's disease. J Intern Med 1998;243:367-72.

82. Beales IL. Gastrointestinal involvement in Behçet's syndrome. Am J Gastroenterol 1998;93:2633.

83. Lenz W. A short history of thalidomide embryopathy. Teratology 1988;38:203-15.

84. Collins TF. History and evolution of reproductive and developmental toxicology guidelines. Curr Pharm Des 2006;12:1449-65.

85. Paine MF. Therapeutic disasters that hastened safety testing of new drugs. Clin Pharmacol Ther 2017;101:430-4.

86. Singhal S, Mehta J, Desikan R, Ayers D, Roberson P, Eddlemon P, et al. Antitumor activity of thalidomide in refractory multiple myeloma. N Engl J Med 1999;341: 1565-71.

87. Hamza MH. Treatment of Behçet's disease with thalidomide. Clin Rheumatol 1986;5:365-71.

88. Gutiérrez-Rodríguez O. Thalidomide. A promising new treatment for rheumatoid arthritis. Arthritis Rheum 1984;27:1118-21.

89. Hamuryudan V, Mat C, Saip S, Ozyazgan Y, Siva A, Yurdakul S, et al. Thalidomide in the treatment of the mucocutaneous lesions of the Behçet syndrome. A randomized, double-blind, placebo-controlled trial. Ann Intern Med 1998;128:443-50.

90. Yasui K, Uchida N, Akazawa Y, Nakamura S, Minami I, Amano $Y$, et al. Thalidomide for treatment of intestinal involvement of juvenile-onset Behçet disease. Inflamm Bowel Dis 2008;14:396-400.

91. Sayarlioglu M, Kotan MC, Topcu N, Bayram I, Arslanturk $\mathrm{H}$, Gul A. Treatment of recurrent perforating intestinal ulcers with thalidomide in Behçet's disease. Ann Pharmacother 2004;38:808-11.

92. Lee HJ, Cheon JH, Lee KJ, Jang HW, Jung KS, Jung ES, et al. Clinical experience of thalidomide in the treatment of Korean patients with intestinal BehcHet's disease: pilot experience in a single center. Intest Res 2010;8:63-9.

93. Bariol C, Meagher AP, Vickers CR, Byrnes DJ, Edwards PD, Hing M, et al. Early studies on the safety and efficacy of thalidomide for symptomatic inflammatory bowel disease. J Gastroenterol Hepatol 2002;17:135-9.

94. Moreira AL, Sampaio EP, Zmuidzinas A, Frindt P, Smith KA, Kaplan G. Thalidomide exerts its inhibitory action on tumor necrosis factor alpha by enhancing mRNA degradation. J Exp Med 1993;177:1675-80.

95. Hatemi I, Hatemi G, Pamuk ON, Erzin Y, Celik AF. TNF-alpha antagonists and thalidomide for the management of gastrointestinal Behçet's syndrome refractory to the conventional treatment modalities: a case series and 
review of the literature. Clin Exp Rheumatol 2015;33(6 Suppl 94):S129-37.

96. Frassanito MA, Dammacco R, Cafforio P, Dammacco F. Th1 polarization of the immune response in Behçet's disease: a putative pathogenetic role of interleukin-12. Arthritis Rheum 1999;42:1967-74.

97. Gül A. Behçet's disease: an update on the pathogenesis. Clin Exp Rheumatol 2001;19(5 Suppl 24):S6-12.

98. Lee CK, Kim HJ. Pathogenesis and treatment of intestinal Behçet's disease. Korean J Gastroenterol 2007;50:3-8.

99. Hassard PV, Binder SW, Nelson V, Vasiliauskas EA. Anti-tumor necrosis factor monoclonal antibody therapy for gastrointestinal Behçet's disease: a case report. Gastroenterology 2001;120:995-9.

100. Lee JH, Kim TN, Choi ST, Jang BI, Shin KC, Lee SB, et al. Remission of intestinal Behçet's disease treated with anti-tumor necrosis factor alpha monoclonal antibody (Infliximab). Korean J Intern Med 2007;22:24-7.

101. Naganuma M, Sakuraba A, Hisamatsu T, Ochiai $H$, Hasegawa $\mathrm{H}$, Ogata $\mathrm{H}$, et al. Efficacy of infliximab for induction and maintenance of remission in intestinal Behçet's disease. Inflamm Bowel Dis 2008;14:1259-64.

102. Lee JH, Cheon JH, Jeon SW, Ye BD, Yang SK, Kim YH, et al. Efficacy of infliximab in intestinal Behçet's disease: a Korean multicenter retrospective study. Inflamm Bowel Dis 2013;19:1833-8.

103. Hibi T, Hirohata S, Kikuchi H, Tateishi U, Sato N, Ozaki K, et al. Infliximab therapy for intestinal, neurological, and vascular involvement in Behcet disease: efficacy, safety, and pharmacokinetics in a multicenter, prospective, open-label, single-arm phase 3 study. Medicine (Baltimore) 2016;95:e3863.

104. Ooi CJ, Hilmi I, Banerjee R, Chuah SW, Ng SC, Wei SC, et al.; Asia-Pacific Association of Gastroenterology (APAGE) Working Group on Inflammatory Bowel Disease and Asian
Organization for Crohn's and Colitis. Best practices on immunomodulators and biologic agents for ulcerative colitis and Crohn's disease in Asia. J Gastroenterol Hepatol 2019; 34:1296-315.

105. Tanida S, Inoue N, Kobayashi K, Naganuma M, Hirai F, Iizuka B, et al. Adalimumab for the treatment of Japanese patients with intestinal Behçet's disease. Clin Gastroenterol Hepatol 2015;13:940-8.e3.

106. De Cassan C, De Vroey B, Dussault C, Hachulla E, Buche $\mathrm{S}$, Colombel JF. Successful treatment with adalimumab in a familial case of gastrointestinal Behcet's disease. J Crohns Colitis 2011;5:364-8.

107. Kimura M, Tsuji Y, Iwai M, Inagaki M, Madian A, Yoshino $\mathrm{T}$, et al. Usefulness of adalimumab for treating a case of intestinal Behçet's disease with trisomy 8 myelodysplastic syndrome. Intest Res 2015;13:166-9.

108. Inoue N, Kobayashi K, Naganuma M, Hirai F, Ozawa M, Arikan D, et al. Long-term safety and efficacy of adalimumab for intestinal Behçet's disease in the open label study following a phase 3 clinical trial. Intest Res 2017;15: 395-401.

109. Vitale A, Emmi G, Lopalco G, Gentileschi S, Silvestri E, Fabiani C, et al. Adalimumab effectiveness in Behçet's disease: short and long-term data from a multicenter retrospective observational study. Clin Rheumatol 2017;36: 451-5.

110. Miyagawa I, Nakano K, Iwata S, Nakayamada S, Saito K, Hanami K, et al. Comparative study of corticosteroid monotherapy, and TNF inhibitors with or without corticosteroid in patients with refractory entero-Behcet's disease. Arthritis Res Ther 2019;21:151.

111. Sugimura N, Mizoshita T, Sugiyama T, Togawa S, Miyaki $\mathrm{T}$, Suzuki T, et al. Real-world efficacy of adalimumab and infliximab for refractory intestinal Behçet's disease. Dig Liver Dis 2019;51:967-71. 